\section{ON OPTICAL PHENOMENA PRODUCED BY CRYSTALS SUBMITTED TO CIRCULARLY POLARISED LIGHT*}

O $\mathrm{N}$ a former occasion I exhibited some phenomena depending upon circular, or, as it was then also called, successive polarisation, and in particular I adopted and explained a method for producing circularly polarised light devised by Sir Charles Wheatstone. I propose on the present occasion to pursue the subject into some of its ulterior consequences. In terms of the wave theory, light is said to be circularly polarised when the vibrations are circular, as distinguished from plane polarisation, when they are rectilinear. And further, it is known from mechanical principles that a circular vibration may always be produced by the combination of two rectilinear vibrations, the amplitudes or extents of which are equal, and whereof one is in advance or in rear of the other by one or by any odd number of quarter-wave lengths. In the former of these cases the circular motion will take place in one direction, say right-handed, in the latter in the opposite, say left-handed. The contrivance used for producing circular polarisation this evening is known by the name of a "quarter undulation plate," and consists of a plate of mica split to such a thickness that one of the two rays into which plane polarised light is divided on entering it is retarded by an odd number of quarter-wave lengths behind the other.

The optical phenomena produced by crystals when submitled to polarised light are usually divided into two classes, viz. (I) those arising from the use of parallel light, and consisting of broad sheets of colour; and (2) those due to convergent light, and consisting of the rings and brushes, the general character of which is well known. I propose to take a few specimens from each class, and to examine the modifications which the known phenomena undergo when the light is both polarised and analysed circularly, i.e., when one quarter-undulation plate is interposed between the polariser (Nicol's pri:m) and the crystal to be examined, and the second between the crystal and the analy ser (Nicol's prism).

In the first place, it is known that if a plate of selenite be placed in an ordiniry apparatus when the polariser ard analyser are either parallel or crossed, there are four positions at $90^{\circ}$ apart in which the plate will produce colour; and further, that if the analyser be turned through $90^{\circ}$ the same result will be obtained, except that the colour will be complementary to that first seen. The intensity of the light at any given point is then given by the form :la :

$$
\cos ^{2} s-\sin .2 i \sin .2(i-s) \sin .^{2} \frac{\theta}{2}
$$

where $i$ and $s$ are the angles made with the original plane of polarisation by the principal sections of the crystal and of the analyser respectively, and $\theta$ is the retardation.

If, however, the two quarter-undulation plates (say the plates $A$ and $B$ ) be introduced, the light undergoes the following processes:-First, it is plane polarise 2 by the polariser; secondly, the plate A being placed so that its axis is inclined at $\pm 45^{\circ}$ to the original plane of polarisation, the light undergoes right or left-handed circulat polarisation, and in that condition falls upon the crystal; thirdly, in their passage through the crystal $\mathrm{C}$ the rays are each clivided into two, whose vibrations are at right angles to one another, and whereof one is retarded in proportion to the thickness of $\mathrm{C}$; fourthly, the plate $\mathrm{B}$ being placed so that its axis is parallel or perpendicular to that of $A$, each of these sets of rays is circularly polarised, one set righthanded, and the other set left-handed; fifthly, these two oppositely circularly polarised sets of rays combine, according to known mechanicallaws, on emerging from $B$ into plane rays, in which the planes of polarisation of the different colours of the spectrum are turned through different angles. Hence finally by turning the analyser round we shall cross these various planes in turn and successively extinguish the different colours, leaving the complementary colours visible. The system of plates $\mathrm{A} C \mathrm{C} \mathrm{B}$ consequently' acts in this respect like quartz. It is, however, to be observed that if the plate $B$ be turned from one of the two proposed positions to the other, the clirections of motion in the two emergent circulariy polarised rays, and consequently the planes of polarisation of the different colours, will be reversed; in other words, with the plate $B$ in one position we shall imitate a right-handed, with the

* Lecture delivered at the Roral Institution of Great Briţain, May 3, $r 872$, by W. Spottiswoode, Treas, R.S. plate $\mathrm{B}$ in the other a left-handed, quartz. The intensity of the light at any point is then given by the formula : -

$$
\begin{aligned}
& \sin ^{2} \frac{\theta}{2} \text { for one position, } \\
& \cos ^{2} \frac{\theta}{2} \text { for the other. }
\end{aligned}
$$

Again, if the plates $\mathrm{A} \mathrm{B}$ retaining either of the positions before indicated, the crystal $\mathrm{C}$ be turned round in its own plane then, since the light emerging from $A$ and $B$ is circularly polarised, it has lost all trace of direction with reference to the positions of the polariser and analyser, and consequently no change of tint will be observed. The same is abundantly clear from the formula written above, because the only term it contains depends upon the retardation within the crystal C. This experiment was made by Airy.

If the plates A and B have their axes directed $45^{\circ}$ on either side of the axis of $\mathrm{C}$, and the three plates be turned round as one piece, the colour will remain unchanged; while, if the analyser be turned, we have the colours shown in the regular order. If the plates $A$ and $B$ have their axes directed at $45^{\circ}$ on the same side of the axis of $\mathrm{C}$, and the pieces be turned round bodily as before, the colours change in the same order as above, and go through their cycle once in every $90^{\circ}$ of rotation; and if the analyser be turned in the same direction the colours change, but in the reverse order. The explanation of this is to be found in the fact that when the plates $A$ and $B$ are crossed, the retarda. tion due by $\mathrm{A}$ is compensated by that due to $\mathrm{B}$; so that the only effective retardation is that due to the crystal $C$. But upon this depends the rotation of the plane of polarisation; if, therefore, the polariser and analyser remain fixed, the colour will remain unaltered. When the plates $A$ and $B$ have their axes parallel, there is no compensation, and the colour will consequently change. This experiment was made by Fresnel. The mathematical expressions for the intensity of the light in the two cases respectively are :-

$$
\cos ^{2}\left(j+i+\frac{\theta}{2}\right) \text {, and } \cos ^{2}\left(j-i-\frac{\theta}{2}\right) \text {, }
$$

where $i$ is the angle made by the principal sections of $A$ with that of the polariser, and $j$ that of the principal section of $\mathrm{B}$ with that of the analyser. The first expression is obviously unchanged when the angle between the polariser and analyser, viz. $\frac{\pi}{2}+i+j$, is unchanged.

It should be added that the rotation of the plane of polarisation, and consequently also the sequence of tints, does not follow exactly the same law in the above cases as in quart $z$.

We now come to the case of convergent light, that is, to the phenomena of crystal rings. And let us examine the effects produced by the same arrangement as before, viz., two quarterundulation plates, A, B, one in front and one behind the crystal C. To quote from Mr. Airy :- "The first thing that strikes us in this combination is that there is nothing, except in the crystal, that has any respect to sides. For the only incident light is circularly polarised; the only light allowed to emerge is circu. lariy polarised. The appearance, therefore, of the coloured rings, \&c., must be such as conveys no trace of any plane of polarisation, and must not vary as the crystal is turned round. In the common exhibition of the coloured rings the principal trace of the planes of polarisation is in the uncoloured brushes. In uniaxal crystals they form an eight-rayed star, composed of two square crosses, inclined at an angle equal to that between the planes of polarisation, every ray of which separates complementary rings. In biaxal crystals they compose two pairs of rectangular hyperbolas, the angle between whose asymptotes is the same as that between the planes of polarisation, and whose branches divide complementary rings. The two crosses or two sets of hyperbolas unite when the planes of polarisation are parallel or ferpendicular. But in the case under consideration the rings exhibited by crystals will not be traversed by any brushes. Uniaxal crystals will exhibit circular rings without a cross; and biaxal crystals will exhibit complete lemniscates, without any interruption from curved brushes." And it is further to be noticed, as the formula given above indicates, that the centres of the rings will be bright or dark according as the analyser stands at $0^{\circ}$ or $90^{\circ}$.

To pursue this matter further. Suppose that, the arrangements remaining otherwise as before, the analyser be turned round; then in any position intermediate to $0^{\circ}$ and $90^{\circ}$ the rings 
will be contracted and extended in opposite quadrants until at $45^{\circ}$ they are divided by two diagonals, on each side of which the colours are complementary. Beyond $45^{\circ}$ the rings begin to coalesce, until at $90^{\circ}$ the four quadrants coincide again. During this movement the centre has changed from bright to dark. If the motion of the analyser be reversed, the quadrants which before contracted now expand, and vice versâ. Again, if the crvstal (say positive) be replaced by another (say negative), the effect on the quadrants of the ring; will be reversed. This method of examination therefore afforus a test of the character, positive or negative, of a crystal.

A similar process applies to biaxal crystals; but in this case the diagonals interrupting the rings are replacel by a pair of rectangular hyperbolas, on either side of which the rings $\mathrm{expand}$ or contract, and the effect is reversed either by reversing the motion of the analyser, or by replacing a positive by a negative crystal, or vice versa. The experiment may then be made in biaxal crystals, by turning the analyser slightly to the ritht or to the left, and observing whether the rings advance towaris or recede from one another in the centre of the field. In particular, if, polariser and analyser being parallel, the plate $A$ have its axis in a N.E. direction to a person looking through the analyser, the plate B its axis in a N.W. direction, and the crystal be so placed that the line joining the optic axes be $\mathrm{N}$.S., then on turning the analyser to the right the rings will advance to one another if the crystal be negative, and recede if it be positive. The ma'hematical expression for the intensity of the light at any point $P$ is in this case

$$
\frac{1}{2}(I+\sin .2 j \cos . \theta+\sin .2 b \cos .2 j \sin . \theta) \text {, }
$$

where $b$ is the angle between the principal section of $C$ through $\mathrm{P}$ and the principal section of $\mathrm{B}$, and $j$ the angle between the principal sections of $B$ and the analyser. This shows that when the polariser and analyser are parallel or crossed at $0^{\circ}$ or $90^{\circ}$, and consequently $j=45^{\circ}$ or $135^{\circ}$, the expression is independent of $b$, i.e., the intensily is the same throughout circles about the centre, but that when the polariser and analy ser are crossed we have an expression of the form

$$
\frac{1}{2}(1 \pm \sin 2 b \sin \theta)
$$

the sign of the second term d pending upon the direstion in which the analyser has been turned, and al so upo.r the sign of $\theta$, that is, upon the character (positive or negative) of the crys:al.

The dispersion of the planes of polarisation effected by the passage of plane polarised light through a plute of quartz cut perpendicular to the axis may be rendered visible by interposing such a plate of quartz between the polariser and a uniaxal or biaxal crystal, when the analyser is at $90^{\circ}$, i.e., when dark brushes are formed. In this case the brushes ce.se to be black, and are tinged throughout with colour. The analyser must, hiswever, be turned back or forward, according as the quartz be right-handed or left-handed, in order that ic may cross in succes. sion the planes of polarisation of the different coloured rays, and so produce the most vivid effects. The dispersion of the brushes i:y a plate of quartz may, however, be studied by employing an auditional polariser and quartz plate between the source of light and the whole system previously used. By turning this polariser round we extinguish each ray of the spectrum in iurn, and tiut the whole field with the complementary colour. The brushes will then appear to revolve about their centre; as the tints vary continuously from one end of the spectrum t.) the other. If the polariser be turned still farther round, the tints which had changed continuously from red to violet, or vice versî, change suddenly from violet to red, or vice versiî, and the brushes jump suddenly back to their original position.

This last optical arrangement may be employed to examine the more important phenomena of the dispersion of the optic axes produced, not by a quartz plate between the u:ual polariser and crystal, but by certain biaxal crystals themselves.

\section{$B O T A N Y$}

\section{The Leaves of Drosera}

IN a recent note to the Paris Academy of Sciences, $M$. Ziegler writes as follows :-

The hairs on the leaves of Droseras exude at their extremity a small drop of glue, by which insects are caught. Whenever an insect becomes attached, the exterior threads bend over it, covering it like the fingers of a hand, and do not straighten again till some days after, when a fresh drop exudes for a fresh prey.
In studying these remarkable plants, I noticed that all the albuminoid animal substances, if held for a moment between the fingers, acquired the property of making the hairs of the Drosera contract. I also observed that such substances, when they had not been in contact with a living animal, had no visible action on the hairs. This shows that the simple contact of the fingers communicates to inert animal substances a property which they did not possess before.

These same animal substances thus prepared lost this singular property when they were moistened several times with distilled water, and dried each time in a water-bath. This is a convenient mode of preparing the substances for experiment.

The contraction of the hairs is not caused by animal heat, which the fingers may have communicated to the animal sub. stances, for the hairs contracted equally when the substance hat been cooled before placing it on the leaf.

The perspiration of the fingers cannot affect the phenomenon, for the property can be communicated to animal substances acrusi fine waxed paper. And the result is not affected if the substances are first covered with a coating of wax, thus preventing any chemical action of solub!e matters which the animal substances may contain.

A living animal thus communicaling by simple contact new physical properties to an inert body, it was important to know whether, by increasing the amount of transmission, we should observe any change in the vital state of the animal. Some rabbits were enclosed in light wooden cages. These were of such a size that their sides were always in contact with the hairs of tl e animal at one part or other. To the outside of the cage were atrached bigs of cloth or paper, containing (for each cage) two kilogrammes of dried serum (albumen from blood). Other rabbits were placed in exactly similar cages, but wilhout the albumen. Their food consisted of 25 grammes of hulled oats every twenty four hours, with cabbage leaves at discretion.

At the end of some days, the rabbits that had been in contact with the a bumen became diabetic in a high degree (thoug $h$ withour saccharine matter) ; the urine was given in normal quantity, but the loss in ammoniaco-magnesian phosphate was very great, and these rabbits deteriorated and lost weight. The other rabbits, which had not been in contact with albumen, remained in their normal state, and even gained weight.

It was interesting to ascertain if the avidity of the Drosera for insects was insatiable, and to find what would be the effect on it of increasing the contact of a living animal. Some dozens were accordingly placed, with a small clod of earth and sufficient water, in light platinum capsules. These capsu'es were each placed in a sheath containing blood aibumen, which had previousiy been held for half an hour in the hand. At the end of twenty-four hours all these Droseras had become quite insensible to insects and to organic animal bodies modified by living contact. The properties of these plants were reversed, and strange to say, their hairs were found to contract under the influence of organic mat ters which had previously been in contact with paper packets (of double or triple envelope) containing sulphate of quinine. Organic matters influenced in this purely physical manner by sulphate of quinine have no contractile action on the hairs of the Drosera in their normal state. The plants whose physical properties have been reversed thy the influence of aluumen in the above way, could be restored to their norinal state by placing them for twenty-four hours with the platinum capsule on a packet of sulphate of quinine. This method may be adopted whenever, from any cause, the leaves have become insensible to insect;. In every case the contraction of the hairs is always slow; it commences visibly in about a quarter of an hour, and is often not complete till after several hours.

Among vegetable matters seeds only are impressible in the way referred to, and the experiments mentioned (which were made with albuminoid animal substances) may be repeated with these.

\section{Nature of Diatoms}

IN a recent essay by Prof. Adolf Weiss, of Lemburg (" $\mathrm{Zum}$ Baue und der Natur der Diatomaceen "), it appears to be demonstrated that the siliceous investment of these little plants has cellulose $f, r$ its base. The silex is infiltrated to a variable extent in the different families, and the mode of its deposition can to a certain extent be ascertained by examination with polarised light. In opposition to the opinion hitherto generally admitted, Prof. Weiss shows that the siliceous coal is capable of 